\title{
AVALIAÇÃO DAS HABILIDADES AUDITIVAS EM CRIANÇAS COM ALTERAÇÕES DE APRENDIZAGEM
}

\section{Evaluating auditory abilities in children with learning disabilities}

\author{
Tatiane Maria Pelitero ${ }^{(1)}$, Alessandra Kerli da Silva Manfredi ${ }^{(2)}$, Andrea Pires Corrêa Schneck ${ }^{(3)}$
}

\section{RESUMO}

Objetivo: comparar o desempenho na Avaliação Simplificada do Processamento Auditivo (ASPA) e no Pediatric Speech Intelligibility Test (PSI), de crianças com alteração de Aprendizagem da Leitura e Escrita e sem este tipo de alteração. Métodos: participaram da pesquisa 28 crianças na faixa etária de 8 a 12 anos, do sexo masculino e feminino. Os participantes foram submetidos ao Teste de Desempenho Escolar (TDE) para a categorização dos grupos de estudo e controle, e, para avaliação das habilidades auditivas foram aplicados a ASPA e o Teste PSI. Resultados: não foi observada associação estatisticamente significante entre o desempenho nos testes de Processamento Auditivo (PA) e o grupo com dificuldades de aprendizagem, apesar de ter sido verificada maior frequência de alterações no grupo de estudo em relação ao grupo controle, em todos os testes. Na ASPA, o teste em que se observou maior número de alterações foi o Teste de Memória Sequencial Verbal, contudo, o Teste de Memória Sequencial Não-verbal foi o que mostrou maior diferença entre os grupos. Conclusão: Não foram encontradas diferenças estatisticamente significantes no desempenho na Avaliação Simplificada do Processamento Auditivo (ASPA) e no Pediatric Speech Intelligibility Test (PSI), das crianças com alteração de Aprendizagem da Leitura e Escrita e sem alteração.

Descritores: Aprendizagem; Transtornos de Aprendizagem; Percepção auditiva; Transtorno da Percepção Auditiva

\section{INTRODUÇÃO}

O aprendizado da leitura e da escrita é de grande importância para $o$ desenvolvimento tanto social quanto emocional do ser humano. No entanto, alguns indivíduos apresentam dificuldades de aprendizagem.

(1) Fonoaudióloga Clínica contratada pelo Setor de Educação da cidade de Santa Cruz das Palmeiras- SP; com Aperfeiçoamento em Disfagia Infantil pelo Hospital das Clínicas da Faculdade de Medicina de Ribeirão Preto da Universidade de São Paulo - USP

(2) Fonoaudióloga contratada do Hospital das Clínicas da Faculdade de Medicina de Ribeirão Preto da Universidade de São Paulo - USP; Mestre em Saúde Pública pela Escola de Enfermagem de Ribeirão Preto-Universidade de São Paulo - USP; Doutora em Ciências Médicas pela Faculdade de Medicina de Ribeirão Preto da Universidade de São Paulo - USP

(3) Fonoaudióloga contratada do Hospital das Clínicas da Faculdade de Medicina de Ribeirão Preto da Universidade de São Paulo; Mestre em Ciências Médicas pela Faculdade de Medicina de Ribeirão Preto- Universidade de São Paulo - USP

Conflito de interesses: inexistente
Numa perspectiva orgânica as dificuldades de aprendizagem são desordens neurológicas que interferem com a recepção, integração e expressão de informação, caracterizando-se, em geral, por uma discrepância acentuada entre o potencial estimado do aluno e sua realização escolar. E numa perspectiva educacional, as dificuldades de aprendizagem refletem uma incapacidade ou impedimento para a aprendizagem da leitura, da escrita, do cálculo ou para a aquisição de aptidões sociais ${ }^{1}$.

Dificuldade de Aprendizagem Específica é uma inabilidade para a aprendizagem, numa ou mais áreas acadêmicas, nada condizente com o potencial intelectual (inteligência) de um aluno, geralmente na média ou acima desta, entrando em conflito direto com os problemas de aprendizagem generalizados do aluno cujo potencial intelectual é bastante abaixo da média (QI abaixo de 70, quando medido por um teste de inteligência). Neste último caso, o aluno faz aprendizagens e tem realizações consentâneas com o seu potencial, não apresentando, portanto, dificuldades de aprendizagem específicas, mas 
sim outra problemática, comumente designada por deficiência mental ${ }^{2}$.

Apesar das dificuldades de aprendizagem estarem presentes mesmo em crianças com inteligência normal e sem alterações perceptuais, a aquisição da leitura e da escrita engloba vários fatores, tanto biológicos quanto sociais da criança. Alguns fatores sociais que podem interferir no aprendizado se referem ao ambiente escolar e familiar, assim como às habilidades e motivação do próprio aprendiz ${ }^{3}$.

Assim, o aprendizado da leitura e da escrita depende de muitos fatores, de condições internas e externas ao desenvolvimento. As condições internas propiciam a percepção de estímulos e possibilitam uma resposta adequada ${ }^{4}$.

A criança com dificuldades de aprendizagem demonstram certas dificuldades perceptivas quanto à identificação, discriminação e interpretação de estímulos. Os processos primários de percepção sensorial parecem apresentar alterações que podem repercutir em dificuldades quanto à leitura, escrita e cálculo ${ }^{5}$.

Uma percepção de fundamental importância é a percepção auditiva. Em relação à audição, é necessário considerar as alterações periféricas e as de natureza central, estas últimas são avaliadas por meio do exame do Processamento Auditivo ${ }^{4}$.

Processamento Auditivo (PA) são os mecanismos do sistema auditivo responsáveis pelos seguintes fenômenos comportamentais: Localização e Lateralização Sonora; Discriminação Auditiva; Reconhecimento Auditivo; Aspectos Temporais da Audição (Resolução, Mascaramento, Integração e Ordenação Temporal); Performance Auditiva diante de sinal acústico competitivo; e Performance Auditiva com decréscimo do sinal acústico ${ }^{6}$.

O Distúrbio do Processamento Auditivo Central (DPAC) acontece quando algo afeta, de forma adversa, o processamento da informação auditiva. Esse distúrbio faz com que ocorra uma dificuldade na interpretação dos padrões sonoros e pode consequentemente ocasionar prejuízos na compreensão das informações, alterações no comportamento e, em decorrência, o fracasso escolar ${ }^{7}$.

Muitos estudos vêm sendo desenvolvidos a fim de caracterizar um distúrbio do processamento auditivo (DPA). O interessante é que podemos caracterizar o comportamento da pessoa com este problema de forma extremamente semelhante ao de uma pessoa com dificuldades de aprendizagem. As semelhanças referem-se à presença de alterações de linguagem; dificuldades com cálculo, leitura e escrita; dificuldades em memorizar; dificuldades em analisar e sintetizar informações; dificuldades em selecionar a figura-fundo; dificuldades para compreender mensagens; dificuldades de atenção ${ }^{8}$.
As dificuldades de linguagem e acadêmicas são frequentemente associadas à desordem do processamento auditivo (DPA). Alguns indivíduos que apresentam a DPA têm uma probabilidade muito elevada para dificuldades comportamentais, emocionais e sociais. Déficits de comunicação associados às dificuldades de aprendizagem podem ter impacto desfavorável no desenvolvimento da auto-estima e do auto-conceito. A identificação e tratamento precoce da DPA podem potencialmente diminuir a probabilidade de que estes problemas secundários surjam ${ }^{9}$.

Portanto, a avaliação do processamento auditivo em crianças na fase de aprendizado escolar, contribui muito para o diagnóstico precoce do Distúrbio do Processamento Auditivo (DPA) e possibilita uma orientação terapêutica e pedagógica mais adequada ${ }^{10}$.

Visto que, o processamento auditivo vem sendo apontado em muitas pesquisas 4,10,11,12,32,33,34,35,36 como um dos fatores da dificuldade de aprendizagem, o objetivo do presente estudo foi comparar o desempenho na Avaliação Simplificada do Processamento Auditivo (ASPA) e no Pediatric Speech Intelligibility Test (PSI), de crianças com alteração de Aprendizagem da Leitura e Escrita e sem alteração.

\section{METODOS}

Trata-se de um artigo original de pesquisa do qual participaram 28 crianças, sendo 13 do grupo de estudo e 15 do grupo controle. Com idades variando entre 8 e 12 anos, do sexo masculino e feminino, pacientes do Setor de Fonoaudiologia e Otorrinolaringologia do Hospital das Clínicas da Faculdade de Medicina de Ribeirão Preto da Universidade de São Paulo (HCFMRP-USP) e alunos de uma escola pública da cidade de Ribeirão Preto, que se encaixaram no perfil do estudo.

Os participantes foram divididos em dois grupos, de acordo com seu desempenho no Teste de Desempenho Escolar (TDE) ${ }^{13}$, sendo o grupo controle, formado pelas crianças que apresentaram desempenho médio ou superior no teste e o grupo de estudo pelas crianças que obtiveram desempenho inferior em pelo menos um dos subtestes do teste em questão.

O Teste de Desempenho Escolar (TDE) ${ }^{13}$ é um instrumento psicométrico que busca oferecer de forma objetiva uma avaliação das capacidades fundamentais para o desempenho escolar, mais especificamente da escrita, aritmética e leitura. O TDE é composto por três subtestes:

- Escrita: escrita do nome próprio e de palavras isoladas apresentadas sob a forma de ditado; 
- Leitura: reconhecimento de palavras isoladas do contexto.

A parte de aritmética foi excluída já que neste estudo foram consideradas apenas as dificuldades de aprendizagem de leitura e escrita.

Cada um dos subtestes apresenta uma escala de itens em ordem crescente de dificuldade, que foram apresentadas ao examinando independente de sua série, uma vez que este pode interromper o subteste assim que os itens apresentados em determinado nível da escala forem impossíveis de serem resolvidos pelo examinando ${ }^{13}$.

Ao final da aplicação do teste TDE foram obtidos os escores de cada criança para os testes de leitura e escrita, que foram comparados com tabela padronizada de acordo com a série escolar.

O TDE foi escolhido para avaliação da Aprendizagem da Leitura e da Escrita por se tratar de um Teste já padronizado, de fácil aplicação e análise.

A seleção dos sujeitos do grupo de estudo foi realizada por meio da análise dos prontuários onde foram verificadas informações a respeito do diagnóstico clínico e desenvolvimento neuropsicomotor para descartar problemas neurológicos ou psiquiátricos, alterações na fala, ou síndrome associada. Já a seleção do grupo controle foi realizada na escola, na qual foram encaminhadas, pelos professores, as crianças que não apresentavam alterações evidentes no desenvolvimento da fala e/ou linguagem ou diagnóstico de distúrbios neurológicos e/ou psiquiátricos.

Os critérios de inclusão para ambos os grupos foram: crianças com níveis de audição normais, tendo como padrão de normalidade a tabela de classificação de perdas auditivas segundo Northern e Downs ${ }^{14}$ e presença de curva do tipo A na Imitanciometria ${ }^{15}$; que não possuíam problemas neurológicos ou psiquiátricos, alterações na fala, ou síndrome associada. No grupo controle foram incluídas as crianças que apresentaram desempenho médio ou superior no teste TDE, ou seja, não apresentaram Dificuldades de Aprendizagem e no grupo de estudo as crianças que apresentaram desempenho Inferior no Teste em questão.

Os equipamentos utilizados para coleta dos dados foram: o audiômetro MIDIMATE 622 da marca Interacoustics que possui dois canais, adequado para a realização do teste de PA; o Imitanciômetro AZ-7 da Madsen; o aparelho de CD player, marca AIWA com saída direta para o audiômetro e CDs ${ }^{16}$ para realização do teste PSI; além de folhas impressas, lápis e borracha para a realização dos testes de aprendizagem.

A coleta dos dados foi realizada no Setor de Fonoaudiologia e Otorrinolaringologia do HCFMRP -
USP e na escola pública da cidade de Ribeirão Preto.

$\mathrm{Na}$ primeira etapa, os sujeitos selecionados que aceitaram participar da pesquisa foram encaminhados para a realização da avaliação clínica médica (otoscopia), onde estava presente o médico Otorrinolaringologista responsável pelo exame, e foi realizada a avaliação audiológica composta pela Audiometria Tonal Liminar, Logoaudiometria e Imitanciometria, para excluir perdas auditivas periféricas.

Posteriormente foram submetidos ao teste TDE para a obtenção do desempenho na aprendizagem da leitura e da escrita e divisão das crianças entre os dois grupos.

Então, a pesquisadora realizou a entrevista com o responsável para aplicação da anamnese prévia aos exames de Processamento Auditivo ${ }^{16} \mathrm{e}$ foram aplicados a Avaliação Simplificada do Processamento Auditivo (ASPA) ${ }^{16}$ e o teste PSI ${ }^{16}$. Os exames do PA e a anamnese foram aplicados após os outros testes, pois foram excluídas do estudo as crianças que apresentaram qualquer alteração nos testes que avaliaram a audição periférica.

É importante ressaltar que apesar de o teste SSI ser o indicado para crianças com idade superior a sete anos ${ }^{16}$, no presente estudo optou-se pelo PSI devido ao fato de serem crianças com habilidade insuficiente de leitura.

Este projeto foi submetido à apreciação e aprovado pelo comitê de Ética do Hospital das Clínicas da Faculdade de Medicina de Ribeirão Preto- Universidade de São Paulo (HCFMRP-USP) no Processo HCRP no 10159/2006. Sendo que os responsáveis por todas as crianças que participaram da pesquisa assinaram ao "Termo de Consentimento Livre e Esclarecido" conforme a Resolução 196/96.

Após aplicação dos testes foi feita a análise dos resultados para cada exame aplicado:

O Teste IPRF foi analisado em função da porcentagem de acertos de cada criança, de acordo com o proposto por Katz ${ }^{17}$.

$\mathrm{Na}$ Imitanciometria foram considerados normais os indivíduos que apresentaram curva do tipo A bilateralmente, e para o Teste do Reflexo Acústico foi considerado como padrão de normalidade limiares de $70 \mathrm{~dB}$ a $100 \mathrm{~dB}^{18}$.

$\mathrm{Na}$ anamnese foi efetuado o cálculo das frequências das queixas mais relevantes em ambos os grupos.

Para a Avaliação Simplificada do Processamento Auditivo e o Teste PSI foram analisados os desempenhos dos participantes de acordo com tabela já padronizada ${ }^{16}$.

E no Teste de Desempenho Escolar (TDE), os Escores Brutos (EB) de cada subteste foram 
convertidos por meio de uma tabela na classificação: superior, médio, inferior para cada série escolar, podendo assim ser utilizado na comparação do desempenho de um indivíduo para outro ${ }^{13}$. Obtiveram desempenho superior e médio nos testes as crianças que apresentaram desempenho igual ou superior ao esperado para a idade e série escolar que frequentam.

Então, para atingir o objetivo proposto foi utilizado o Teste Estatístico de Fisher por meio do software SAS $9.0{ }^{19}$ e foi fixado um nível de significância de 0,05 ou $5 \%{ }^{20}$.

\section{RESULTADOS}

No grupo de estudo foi encontrado um predomínio de indivíduos do sexo masculino $(84,6 \%)$ e no grupo controle do sexo feminino $(53,4 \%)$.
A média de idades para ambos os grupos foi entre 9 e 10 anos. E a maioria dos sujeitos avaliados estava cursando as séries escolares iniciais, predominando os sujeitos que cursavam a $3^{\text {a }}$ série $(38,5 \%$ no grupo de estudo e $46,6 \%$ no grupo controle).

Todas as crianças apresentaram o nível de audição dentro dos padrões de normalidade, Curva Timpanométrica do Tipo A bilateralmente e presença dos Reflexos contra e ipsilaterais com valores abaixo de $100 \mathrm{~dB}$.

A tabela 1 apresenta o desempenho das crianças no Teste TDE por grupo. No grupo de estudo $92,3 \%$ dos participantes da pesquisa obtiveram desempenho inferior e $7,7 \%$ desempenho médio no subteste de escrita e mesmo valor foi encontrado no subteste de leitura. É importante citar que os $7,7 \%$ correspondem a um único indivíduo que não é o mesmo em ambos os subtestes.

Tabela 1 - Desempenho no Teste TDE por grupo

\begin{tabular}{lcccc}
\hline & $\begin{array}{c}\text { Grupo Estudo } \\
\text { (TDE - Escrita) }\end{array}$ & $\begin{array}{c}\text { Grupo Estudo } \\
\text { (TDE - Leitura) }\end{array}$ & $\begin{array}{c}\text { Grupo Controle } \\
\text { (TDE - Escrita) }\end{array}$ & $\begin{array}{c}\text { Grupo Controle } \\
\text { (TDE - Leitura) }\end{array}$ \\
\hline Desemp. Inf. & $92,3 \%$ & $92,3 \%$ & - & \\
Desemp. Med. & $7,7 \%$ & $7,7 \%$ & $86,6 \%$ & $93,3 \%$ \\
Desemp. Sup. & & & $13,4 \%$ & $6,7 \%$ \\
\hline
\end{tabular}

No grupo controle $86,6 \%$ dos indivíduos avaliados apresentaram desempenho médio e 13,4\% desempenho superior para o subteste de Escrita, já no subteste de leitura $93,3 \%$ obtiveram desempenho médio e $6,7 \%$ desempenho superior. Sendo que apenas um sujeito apresentou desempenho superior em ambos os subtestes.
Na tabela 2 observam-se as frequências das queixas mais relevantes por grupo. Foi possível notar um maior número de queixas quanto à dificuldade em ouvir em ambiente ruidoso, desatenção, dificuldade em compreender a conversação, dificuldades de memória e história de otites de repetição no grupo de estudo em relação ao grupo controle.

Tabela 2 - Frequência das queixas mais relevantes por grupo

\begin{tabular}{lcc}
\hline Questões & Grupo Estudo & Grupo Controle \\
\hline Ouve bem em ambiente silencioso? & $100 \%$ & $100 \%$ \\
Ouve bem em ambiente ruidoso? & $63,60 \%$ & $80 \%$ \\
Localiza o som? & $90,90 \%$ & $86,60 \%$ \\
É desatento? & $90,90 \%$ & $40 \%$ \\
É agitado? & $63,60 \%$ & $60 \%$ \\
É muito quieto? & $18,20 \%$ & $13,30 \%$ \\
Compreende bem a conversação? & $54,50 \%$ & $80 \%$ \\
Apresenta facilidade para memorizar nomes? & $36,40 \%$ & $100 \%$ \\
Apresenta facilidade para memorizar lugares? & $36,40 \%$ & $86,60 \%$ \\
Apresenta facilidade para memorizar situações? & $18,20 \%$ & $100 \%$ \\
Apresenta história de otites de repetição? & $36,40 \%$ & $20 \%$ \\
\hline
\end{tabular}


Apesar de não ter sido observada associação estatisticamente significante entre o desempenho na ASPA e o grupo com dificuldades de aprendizagem, observou-se um maior número de alterações no grupo de estudo comparado ao grupo controle.

O teste em que se observou maior número de alterações foi o Teste de Memória Sequencial Verbal, seguido do Teste de Memória Sequencial Não-verbal e do Teste de Localização Sonora. No entanto, o Teste de Memória Sequencial NãoVerbal foi o que mostrou maior diferença entre os grupos, sendo que o grupo controle não apresentou alteração neste teste.

Tabela 3 - Frequências de Alterações na ASPA e p-valores dos cruzamentos das varáveis grupo e desempenho neste teste

\begin{tabular}{lccc}
\hline & $\begin{array}{c}\text { Frequência de alteração } \\
\text { (Grupo estudo) }\end{array}$ & $\begin{array}{c}\text { Frequência de alteração } \\
\text { (Grupo controle) }\end{array}$ & p-valor \\
\hline Localização & 15,38 & 0 & 0,21 \\
MSV & 38,46 & 13,33 & 0,20 \\
MSNV & 23,08 & 0 & 0,09 \\
\hline
\end{tabular}

Teste Estatístico de Fisher $(p<0,05)$

São apresentadas nas tabelas abaixo as frequências das alterações no teste PSI e os p-valores do cruzamento das variáveis grupo e alteração no teste em questão, para ambas as orelhas. Assim como na ASPA, pode-se notar que não foram observadas associações estatisticamente significantes entre as alterações no teste PSI e o grupo com alterações de aprendizagem, porém o grupo de estudo apresentou também neste teste maior número de alterações do que o grupo controle, sendo que este último não apresentou alterações no teste em questão.

Tabela 4 - Frequências das alterações no teste PSI em ambos os grupos e p-valores do cruzamento das variáveis grupo e desempenho no Teste PSI (Orelha Direita)

\begin{tabular}{lccc}
\hline & $\begin{array}{c}\text { Frequência de alteração } \\
\text { (Grupo estudo) }\end{array}$ & $\begin{array}{c}\text { Frequência de alteração } \\
\text { (Grupo controle) }\end{array}$ & p-valor \\
\hline MCC-S/R 0 & 7,69 & 0 & 0,46 \\
MCC-S/R -40 & 7,69 & 0 & 0,46 \\
MCI-S/R 0 & 15,38 & 0 & 0,21 \\
MCI-S/R -10 & 15,38 & 0 & 0,21 \\
MCI-S/R -15 & 15,38 & 0 & 0,21 \\
\hline
\end{tabular}

Teste Estatístico de Fisher $(p<0,05)$

Tabela 5 - Frequências das alterações no teste PSI em ambos os grupos e p-valores do cruzamento das variáveis grupo e desempenho no Teste PSI (Orelha Esquerda)

\begin{tabular}{lccc}
\hline & $\begin{array}{c}\text { Frequência de alteração } \\
\text { (Grupo estudo) }\end{array}$ & $\begin{array}{c}\text { Frequência de alteração } \\
\text { (Grupo controle) }\end{array}$ & p-valor \\
\hline MCC-S/R 0 & 15,38 & 0 & 0,21 \\
MCC-S/R -40 & 7,69 & 0 & 0,46 \\
MCl-S/R 0 & 15,38 & 0 & 0,21 \\
MCI-S/R -10 & 15,38 & 0 & 0,21 \\
MCI-S/R -15 & 15,38 & 0 & 0,21 \\
\hline
\end{tabular}

Teste Estatístico de Fisher $(p<0,05)$ 


\section{DISCUSSÃO}

A Alteração do Processamento Auditivo vem sendo apontada em muitos estudos 4,5,10-12,21,22,23 como uma das causas das Dificuldades de Aprendizagem da Leitura e da Escrita. Logo, a audiologia tem reconhecido que não somente as alterações condutivas e neurossensoriais determinam distúrbios de linguagem e aprendizagem, valorizando também o Processamento Auditivo ${ }^{24}$.

No presente estudo, apesar de não ter sido encontrada diferença estatisticamente significante entre os dois grupos, observou-se uma frequência maior de alterações no grupo de estudo, em relação ao grupo controle, em todos os testes de processamento auditivo. $\mathrm{O}$ que mostra que as crianças com dificuldades de aprendizagem foram as que apresentam maior número de alterações do PA.

Observou-se um predomínio de dificuldades de Aprendizagem em crianças do sexo masculino, com idade entre 9 e 10 anos e estudantes da $3^{\text {a }}$ série. Corroborando os estudos de outros autores ${ }^{5,9}$ que encontraram um predomínio de alterações em crianças do sexo masculino, na faixa etária de 8 a 10 anos e estudantes da $3^{\underline{a}}$ série.

O maior número de crianças com queixas escolares na terceira série, talvez, se deva ao fato de que as exigências escolares tendem a ser gradativamente mais complexas a partir deste nível e também porque as habilidades de análise, síntese, memorização, raciocínio lógico, entre outras, tornam-se mais complexas, exigindo um desempenho melhor dos alunos ${ }^{10}$.

Em anamnese, observaram-se um maior número de queixas quanto à dificuldade em ouvir em ambiente ruidoso, desatenção, dificuldade em compreender a conversação, dificuldades de memória e história de otites de repetição no grupo de estudo em relação ao grupo controle.

Nos casos de DPA, é uma questão significante saber como a otite média pode ter maiores consequências no desenvolvimento das habilidades auditivas e outras funções relacionadas. Logo, devido à grande influência da otite média, é importante determinar a ocorrência de patologias relacionadas com o ouvido médio durante a primeira infância ${ }^{25}$.

São manifestações da DPA, dentre outras: Dificuldades na aprendizagem da leitura e escrita; Dificuldade em manter atenção a estímulos puramente auditivos, pedindo constantes repetições; Discriminação dos sons da fala prejudicada na presença ou não de estímulos simultâneos ou competitivos; Falha de memorização das mensagens ouvidas; Dificuldades na organização e sequencialização de estímulos verbais e não-verbais. Sendo que esta última característica pode ser avaliada pela ASPA ${ }^{26}$.

$\mathrm{Na}$ ASPA, o teste em que se observou maior número de alterações foi o Teste de Memória Sequencial Verbal, seguido do Teste de Memória Sequencial Não-verbal e do Teste de Localização Sonora. No entanto, o Teste de Memória Sequencial Não-verbal foi o que mostrou maior diferença entre os grupos, sendo que o grupo controle não apresentou alteração neste teste.

Estes resultados foram semelhantes aos encontrados em outros trabalhos $27,31,32$, realizados com crianças que apresentavam dificuldades na Aprendizagem da Leitura e da Escrita e foram diferentes dos encontrados por outros autores ${ }^{4,5,10,36}$ que observaram maior número de alterações no Teste de Memória Sequencial Não-verbal, e também em uma outra pesquisa em que a habilidade mais prejudicada foi a de Localização Sonora, seguida da Memória Sequencial Não-verbal e da Memória Sequencial Verbal ${ }^{21}$.

A discrepância entre os dados observados na literatura parece sugerir a importância da utilização das três provas para triagem do PA, pois é a observação conjunta do desempenho da criança nas três tarefas que leva a identificar uma alteração do PA ${ }^{5}$.

Quanto ao Teste PSI observou-se um número reduzido de crianças com alterações, todas elas do grupo de estudo. Sendo que, das crianças que falharam no Teste, duas apresentaram alteração em praticamente todas as condições e uma em apenas uma das condições de aplicação do mesmo.

Outros autores ${ }^{28}$ observaram que o teste PSI$\mathrm{MCl}$ foi adequado para diferenciar os dois grupos estudados, apenas na orelha direita na relação fala/ ruído 0 e -10, havendo nesta situação, uma associação entre os resultados obtidos no PSI-MCI e o grupo com distúrbio de aprendizagem. Os autores associaram este resultado à faixa etária das crianças que participaram da pesquisa (9-11 anos), já que o teste é indicado para crianças de até sete anos.

No presente estudo o teste PSI também foi aplicado em crianças com idade superior à indicada, pois tratam-se de crianças com habilidade insuficiente de leitura, assim como no trabalho citado acima.

Em outra pesquisa ${ }^{29}$ realizada com crianças na faixa etária entre 5 e 7 anos, foi encontrada diferença significante no desempenho neste teste entre o grupo normal e o grupo com dificuldades de aprendizagem em todas as condições de aplicação do mesmo.

Para diagnosticar a DPA, o audiologista precisa aplicar uma série de testes em cabina acústica. Depois de realizado o diagnóstico deve-se 
determinar a natureza do distúrbio. Pois existem diversos tipos de distúrbios do PA, e, como cada criança possui características individuais, a DPA pode manifestar-se de formas diferentes ${ }^{30}$.

Outro fator importante a ser considerado na avaliação do Processamento Auditivo é a motivação e a atenção das crianças ${ }^{33}$, visto que trata-se de uma bateria de testes complexos, o que torna muitas vezes sua realização uma tarefa difícil para a criança, podendo influenciar seus resultados.

Como o teste PSI avalia a habilidade de figurafundo, talvez, fosse necessária a aplicação de outros testes para detalhar o comportamento auditivo das crianças em questão. É recomendável, portanto, sempre possível aplicar nas crianças com dificuldades de aprendizagem uma bateria de testes que avaliam várias habilidades auditivas, como resolução temporal, fechamento auditivo, dentre outras.
Apesar de não ter sido encontrada associação estatisticamente significante do desempenho nos testes de Processamento Auditivo e o grupo com alteração na aprendizagem da leitura e da escrita observou-se maior frequência de alterações no grupo de estudo, em relação ao grupo controle, em todos os testes.

\section{CONCLUSÃO}

Não foram encontradas diferenças estatisticamente significantes no desempenho na Avaliação Simplificada do Processamento Auditivo (ASPA) e no Pediatric Speech Intelligibility Test (PSI), das crianças com alteração de Aprendizagem da Leitura e Escrita e sem alteração.

\begin{abstract}
Purpose: to compare performance of children with or without alterations in reading and writing skills acquisition in the Simplified Auditory Processing Test (SAPT) and the Pediatric Speech Intelligibility (PSI) tests. Methods: twenty-eight female and male children aged 8-12 took part in this study. The subjects did the Academic Achievement Test (TDE) in order to be placed in the study group or control group and, for the assessment of hearing abilities, they took the SAPT and the PSI tests. Results: no statistically significant association was found between performances in tests for hearing processing and the group with learning problems although alterations were observed in the study group more frequently than in the control group in all tests. In SAPT, the test which detected more alterations was the test for verbal Sequential Memory; however, the Nonverbal Sequential Memory test was the one which showed more differences between the groups. Conclusion: no statistically significant association between performances were found both by the HPSA and the PSI tests in children with or without alterations in reading and writing skills acquisition.
\end{abstract}

KEYWORDS: Learning; Learning Disorders; Auditory Perception; Auditory Perceptual Disorders

\section{REFERÊNCIAS BIBLIOGRÁFICAS}

1. Correia LM, Martins AP. Dificuldade de aprendizagem: Que são? Como entendê-las?. In: Biblioteca Digital: Coleção Educação. Rio de Janeiro: Porto Editora; 2005.

2. Correia LM. Para uma Definição Portuguesa de Dificuldades de Aprendizagem específicas. Rev. Bras. Educ. 2007; 13(2): 155-72.

3. Felippe ACN, Colafêmina JF. Avaliação simplificada do processamento auditivo e o desempenho em tarefas de leitura - escrita. PróFono Rev Atual. Cient. 2002 Maio/ Ago.; 4(2): 225-34.
4. Silva F, Coelho JC, Ortiz KZ. Correlação entre processamento auditivo central e a produção gráfica em escolares da $3^{a}$ série do $1^{\circ}$ grau. Pró-Fono Rev. Atual. Cient.1999; 11(1): 42-46.

5. Lucca SA, Mancine MS, Dell'Agli BAV. Dificuldade de aprendizagem: contribuições da avaliação neuropsicológica. Pensamento Plural: Revista Científica da UNIFAE. 2008; 2(1): 32-42.

6. ASHA - American Speech-Language-Hearing Association. Central Auditory Processing: current status of research and implications for clinical practice. Am J Audiol. 1996 Jul; 5(2): 41-54.

7. Martins JS, Pinheiro MMC, Blasi HF. A utilização de um software infantil na terapia fonoaudiológica 
de Distúrbio do Processamento Auditivo Central. Rev. Soc. Bras. Fonoaudiol. 2008; 13(4): 398-404

8. Ribas-Guimarães A. Alterações no processamento auditivo central e as dificuldades de aprendizagem. Jornal Brasileiro de Fonoaudiologia 2000; 1(3): $16-20$.

9. ASHA (AMERICAN SPEECH LANGUAGE HEARING ASSOCIATION). (Central) Auditory Processing Disorders, 2005. Disponível em: http:// www.asha.org/members/deskref-journals/deskref/ default. Acesso em 25 de Abril de 2006.

10. Aita ADC, Mesquita CDS, Campos CM, Fukuda MTH, Aita FS. Correlação entre as desordens de processamento auditivo central e queixas de dificuldades escolares. J Bras Fonoaud. 2003 Abr/ Jun; 4(15): 101-7

11. Moura DRV, Feniman MR, Lauris JRP. Teste de Fusão Auditiva-Revisado em crianças com distúrbios de leitura e escrita. J Bras. Fonoaud. 2000 Abr/Maio/Jun.;1(3): 38-43.

12. Neves IF, Schochat E. Maturação do processamento auditivo em crianças com e sem dificuldades escolares. Pró-Fono Rev. Atual. Cient. 2005 Set./Dez.; 17(3): 311-20.

13. Stein LM. TDE-Teste de Desempenho Escolar: manual para aplicação e interpretação. São Paulo: Casa do Psicólogo; 1994.

14. Northern JL, Downs MP. Audição em crianças. 3aㅡ ed. São Paulo: Manole, 1989.

15. Jerger J. Clinical Experience with Impedance Audiometry. In: Arch. Otolaryng., 1970, p. 311.

16. Pereira LD, Schochat E. Processamento Auditivo: Manual de avaliação. São Paulo: Lovise; 1997.

17. Katz, J. Handbook of Clinical Audiology. Baltimore: The Williams \& Wilkins Co., 1972.

18. Carvalho, RMM. Medidas de Imitância Acústica em Crianças. In: Lichtig I, Carvalho RMM. Audição Abordagens Atuais. São Paulo: Pró-Fono; 1997. p.66-87.

19. Frazza MM, Caovilla HH, Munhoz MSL, Silva MLG, Ganança MM. Imitanciometria. In: Munhoz MSL, Caovilla HH, Silva MLG, Ganança MM. Audiologia Clínica: Série Otoneurológica. $1^{\underline{a}}$ ed. São Paulo: Atheneu; 2003. p.85-101

20. SAS Institute Inc., SAS/STAT® User's Guide, Version 9, Cary, NC: SAS Institute Inc., 2003.

21. Pagano M, Gavreau K. Princípios de Bioestatística, $2^{2}$ ed. São Paulo: Pioneira; 2004.

22. Cruz PC, Pereira LD. Comparação do desempenho das habilidades auditivas e de linguagem, em crianças com queixa de dificuldade de aprendizagem. Acta AWHO 1996 Jan./Mar.; 15(1): 21-6.

23. Sauer L, Pereira LD, Ciasca SM, Pestun M, Gerreiro MM. Processamento Auditivo e SPECT em crianças com Dislexia. Arq. Neuro-Psiquiatr. 2006 Mar.; 64(1): 108-11.

24. Murphy CFB, Schochat, E. Influência de Paradigmas Temporais em Testes de Processamento Temporal Auditivo. Pró-Fono Rev. Atual. Cient. 2007 Jul./Setem.; 19(3):

25. Tedesco MRM. Diagnóstico e terapia do aprendizado da leitura e escrita. In: Lopes Filho O, organizador. Tratado de Fonoaudiologia. $1^{\mathrm{a}}$ ed. São Paulo: Rocca; 1997. p.907-24.

26. Katz J, Tillery KL, Mecca F. (Tradução). Uma Introdução ao Processamento Auditivo. In: Lichtig I, Carvalho RMM. Audição: Abordagens Atuais. São Paulo: Pró-Fono; 1997. p.145-72.

27. Alvarez AMMA, Balen AS, Misorelli MIL, Sanchez ML. Processamento Auditivo Central: Proposta de Avaliação e Diagnóstico Diferencial. In: Munhoz MSL, Caovilla HH, Silva MLG, Ganança MM. Audiologia Clínica: Série Otoneurológica. 1aㅡ ed. São Paulo: Atheneu; 2003. p. 103-20.

28. Furbeta TD, Felippe ACN. Avaliação Simplificada do Processamento Auditivo e Dificuldade de LeituraEscrita. Pró-Fono Revista de Atualização Científica. 2005 Jan./Abr.; 17(1): 11-18.

29. Garcia VL, Pereira LD, Fukuda Y. Atenção seletiva: PSI em crianças com distúrbio de aprendizagem. Rev. Bras. Otorrinolaringol. 2007 Maio/Jun.; 73(3): 404-11.

30. Almeida CIR, Lourenço EA, Caetano MHU, Duprat AC. Disfunção auditiva central nas crianças portadoras da Deficiência do Aprendizado. Rev Bras Otorrinolaringol. 1990 Abr/ Jun; 56(2): 64-8.

31. Bellis TJ. Understanding Auditory Processing Disorders in children. ASHA - American SpeechLanguage-Hearing Association [artigo online]. Disponível em: http//www.asha.org/public/hearing/ disorders/understand-apd-hild.htm (25 Abr 2006).

32. Engelmann L, Ferreira MIDC. Avaliação do processamento auditivo em crianças com dificuldades de aprendizagem. Rev Soc Bras Fonoaudiol. 2009; 14(1): 69-74.

33. Ramos CS, Pereira LD. Processamento auditivo e audiometria de altas frequências em escolares de São Paulo. Pró-Fono Rev. Atual. Cient. 2005; 17(2): 153-64.

34. Hurley RM. Auditory processing disorder evaluation: Using a reinforcement paradigm. The ASHA Leader. 2007; 12(15): 5-6.

35. Simon LF, Rossi AG. Triagem do processamento auditivo em escolares de 8 a 10 anos. Psicol. Esc. Educ. dez. 2006; 10(2): 293-304.

36. Lucas PA, Zacare CC, Alves Filho OC, Amantini RCB, Bevilacqua MC, Zaidan E. Scan: perfil de desempenho em crianças de sete e oito anos. Prófono R. Atual. Cient. 2007 Out./Dez; 19(4): 370-73. 
37. Colella-Santos MF, Bragato GR, Martins PMF, Ballarin A. Triagem Auditiva em Escolares de 5 a 10 anos. Rev. CEFAC [artigo online]. 2009 Jul. Disponível em: http://www.scielo.br/scielo.php?script=sci arttext\&pid=S1516-18462009005000037\&lng=en\& nrm=iso. Acesso em 14 Out 2009.

38. Costa-Ferreira MID, Sávio CB. Relação entre transtorno de processamento auditivo e dificuldades na compreensão leitora. Letrônica. 2009 Jul.; 2(1): 26-41.

39. Farias LS, Toniolo IF, Cóser PL. P300: avaliação eletrofisiológica da audição em crianças sem e com repetência escolar. Rev Bras Otorrinolaringol. 2004; 70(2): 194-9.

DOI: 10.1590/S1516-18462010005000062

RECEBIDO EM: 04/02/2009

ACEITO EM: 02/04/2010

Endereço para correspondência:

Tatiane Maria Pelitero

Av. XV de Novembro, no 40 - Centro

Santa Cruz das Palmeiras - SP

CEP: $13650-000$

E-mail: thatyp22@yahoo.com.br 